\title{
Stochastic Geometry Analysis of Error Probability in Interference Limited Wireless Networks
}

\author{
Yamuna Dhungana and Chintha Tellambura \\ Department of Electrical and Computer Engineering \\ University of Alberta, Edmonton, Alberta T6G 2V4, Canada \\ dhungana@ualberta.ca, ct4@ualberta.ca
}

\begin{abstract}
In this paper, we present mathematical frameworks for error performance analysis in interference limited networks such as cellular networks. Due to the increasing irregularity in the spatial deployment of nodes in the emerging heterogeneous cellular networks (HCNs), we employ stochastic geometry approach by abstracting the node locations as a homogeneous Poisson point process (PPP). First, we characterize the average error probability of an intended communication link with a given transmitter-receiver separation, which is subject to interference from these Poisson distributed nodes. More specifically, we develop uniform approximation (UA), which is highly accurate over the whole range of signal-to-interference ratio (SIR) and hence, serve as an alternative to existing complex analytical results. Error probability UAs for both single-antenna and maximal ratio combining (MRC) receivers are derived in this paper. Next, we evaluate the average error probability of any typical user in the network, which is served by the node providing the maximum received power. Mellin-transform based method is proposed in this case, which often yield closed-form solution. An example of BPSK modulation is given in the paper.
\end{abstract}

\section{INTRODUCTION}

In large wireless networks with numerous nodes spatially distributed over very large areas, such as cellular networks, the performance limiting factor is interference rather than noise. The interference is a direct function of the spatial configuration of the network on which wireless propagation characteristics such as path loss, shadowing and multipath fading are dependent upon. As cellular networks emerge towards heterogeneous deployments (low-power nodes such as picos and femtos overlaid with the macro-cellular infrastructure), which are characterized by unplanned/random locations of the nodes, the interference scenario becomes more complex, and computation of spatially averaged performance metrics becomes critical to derive useful design insights. To this end, stochastic geometry has been recently proven to be a powerful mathematical tool [1], [2]. It allows to evaluate the average network behavior over all possible spatial realizations of the nodes, which are abstracted by a suitable spatial point process. Poisson point process (PPP) is the most popular and widely used spatial model for wireless networks due to its analytical tractability and the availability of large class of powerful results and well-established analytical tools [1]-[3].

The spatial distributions of base-stations (BSs) of each tier (e.g., macro, pico, femto) in a multi-tier heterogeneous cellular network $(\mathrm{HCN})$ are modeled as independent PPPs in
[4]-[8]. Coverage probability and/or average achievable rate are analyzed in these work for different BS-user association and interference coordination schemes by using tools from stochastic geometry and point process theory. It was shown in [9] that even for the planned macro tier, the PPP model provides tight lower bounds for performance measures such as coverage and data rate.

Apart from coverage and data rate, the effectiveness of wireless network is also characterized by its reliability, measured with metric such as error probability, which is barely analyzed for HCNs. Few important works towards error probability analysis in the presence of interference from randomly located network nodes are [10]-[13]. The authors in [10], [11] developed a comprehensive framework to characterize the error performance of a given transmitter-receiver link subject to interference from network nodes distributed according to a homogeneous PPP. However, the computation requires MonteCarlo simulations to average over the network interference which is shown to be a stable random variable (RV). Such requirement is eliminated in [12] where the authors derived a single-integral expression for the average error probability. Since no closed-form solution is available for the integral, it has to be evaluated numerically. It is further extended to multiantenna receivers in [13], where the results were obtained in the form of two-fold integral that reduces to single-integral only under special cases.

As an alternative to these semi-analytical solution and complex integral expression, in this paper, we develop a uniform approximation (UA) approach to average error probability analysis of the desired link in Poisson field of interferers. In cellular networks with frequency reuse 1 , which is the main characteristic of next-generation wireless standards such as LTE-Advanced for higher spectral efficiency, the performance is limited by interference, rather than noise. We thus focus on interference-limited scenarios and derive error probability UAs for both single-antenna and maximal ratio combining (MRC) receivers. The approximation is named "uniform" to reflect its excellent accuracy over the whole range of signalto-interference ratio (SIR). We originally proposed the concept of UA for wireless performance analysis in [14], [15].

After deriving UA for the average error probability of a given link with a deterministic transmitter-receiver distance $r_{0}$ in section II, we next evaluate the average error probability of 
any typical user in downlink cellular network with maximum received power based BS-user association in section III. In this case, the transmitter-receiver distance is no longer deterministic. The error performance of downlink cellular networks for shortest distance based BS-user association is analyzed in [16]. The mathematical framework we developed in this paper for interference-limited scenario is however more simple and easily provides insights on important system parameters.

Notations: We denote a 2-dimensional Euclidean space by $\mathbb{R}^{2}$ and Euclidean distance between two points $x, y \in \mathbb{R}^{2}$ by $\|x-y\|$. A real and a circularly symmetric (CS) complex Gaussian RV with mean $m$ and variance $\sigma^{2}$ is denoted by $\mathcal{N}\left(m, \sigma^{2}\right)$ and $\mathcal{C N}\left(m, \sigma^{2}\right)$, respectively. $\mathbb{P}(\cdot), \mathbb{E}[\cdot]$ and $\mathcal{F}_{B}(s)=\int x^{s-1} f_{B}(x) d x$ denote probability measure, statistical expectation and Mellin transform, respectively. $\mathcal{S}(\mu, \beta, \eta)$ denotes a real stable RV with characteristic exponent $\mu$, skew parameter $\beta$ and dispersion $\eta$ [17]. $Q(\cdot)$ denotes the Gaussian Q function, and $|s|$ and $s^{*}$ denote the modulus and conjugate of complex number $s$, respectively.

\section{UNIFORM APPROXIMATION FOR AVERAGE ERROR Probability In POISSON FIELd OF INTERFERERS}

\section{A. System and channel model}

We consider a 2-dimensional network in which a transmitter $S$ (located at $x_{s} \in \mathbb{R}^{2}$ ) intends to communicate with a receiver $D$ (located at the origin $o$ without loss of generality). The distance between $S$ and $D$ is fixed at $\left\|x_{s}-o\right\|=r_{0}$. Other nodes in the network which are transmitting in the same channel as $S$ and thus, interfering with $S-D$ communication, are spatially distributed according to a homogeneous PPP $\Phi=\left\{x_{1}, x_{2}, x_{3}, \ldots\right\}$ of density $\lambda$, where $x_{i} \in \mathbb{R}^{2}$ is the location of the $i$ th interferer. For simplicity of analytical expressions, we assume that the transmissions from interfering nodes are synchronized. Results for asynchronous case can be easily derived and are omitted here for the sake of brevity.

We first consider the case where each node in the network including $S$ and $D$ have single antenna. With multipath fading superimposed on power-law path loss and shadowing, the channel power gain between a transmitter at $x \in \mathbb{R}^{2}$ and the receiver $D$ at the origin can be modeled as

$$
\Omega_{x}=e^{\sigma g_{x}}\left|h_{x}\right|^{2}\|x-o\|^{-\alpha},
$$

where $e^{\sigma g_{x}}$ captures the shadowing effect modeled by lognormal distribution with $g_{x} \sim \mathcal{N}(0,1), \sigma$ is the shadowing standard deviation, $h_{x}=\left|h_{x}\right| \exp \left(j \psi_{x}\right)$ is the complex multipath fading coefficient with $\mathbb{E}\left[\left|h_{x}\right|^{2}\right]=1$, and $\alpha$ is the power loss exponent. In (1), $g_{x}$ and $h_{x}$ are independent RVs.

If the source $S$ transmits with power $P_{0}$ and each interfering node with power $P_{I}$, then according to the channel model (1), the complex received signal at $D$ is given by

$$
Y=\sqrt{P_{0}} \frac{e^{\frac{\sigma}{2} g_{x_{s}}} h_{x_{s}}}{r_{0}^{\alpha / 2}} s_{0}+Z+W,
$$

where

$$
Z=\sqrt{P_{I}} \sum_{x_{i} \in \Phi} \frac{e^{\frac{\sigma}{2} g_{x_{i}}} h_{x_{i}}}{r_{i}^{\alpha / 2}} s_{i}
$$

is the aggregate interference signal, $r_{i}=\left\|x_{i}-o\right\|$ is the distance between the $i$ th interferer at $x_{i}$ and the receiver $D, W \sim \mathcal{C N}\left(0, N_{0}\right)$ is complex Gaussian noise, and $s_{0}=$ $a_{0} \exp \left(j \theta_{0}\right), s_{i}=a_{i} \exp \left(j \theta_{i}\right)$ are the complex modulated symbols transmitted from $S$ and the $i$ th interferer, respectively with $\mathbb{E}\left[\left|s_{0}\right|^{2}\right]=\mathbb{E}\left[\left|s_{i}\right|^{2}\right]=1$. While all the interfering nodes are assumed to be using the same linear modulation scheme, the transmitter $S$ employs an arbitrary linear modulation. We assume each sequence $\left\{g_{x_{i}}\right\},\left\{h_{x_{i}}\right\}$ and $\left\{s_{i}\right\}$ are independent and identically distributed (i.i.d.). If the phase $\psi_{x_{i}}$ of multipath fading coefficient $h_{x_{i}}$ is uniformly distributed in $(0,2 \pi)$, then it is shown in [11] that $Z$ is a CS complex stable RV whose distribution is given by

$$
Z \sim \mathcal{S}_{c}\left(\mu_{z}=\frac{4}{\alpha}, \beta_{z}=0, \eta_{z}\right)^{1}
$$

for $\alpha>2$, where $\eta_{z}=\pi \lambda C_{4 / \alpha}^{-1} e^{2 \sigma^{2} / \alpha^{2}} P_{I}^{2 / \alpha} \mathbb{E}\left[\left|\xi_{i}\right|^{4 / \alpha}\right]$,

$$
C_{x}= \begin{cases}\frac{1-x}{\Gamma(2-x) \cos (\pi x / 2)}, & x \neq 1 \\ \frac{2}{\pi}, & x=1,\end{cases}
$$

and $\xi_{i}=\left|h_{x_{i}}\right| a_{i} \cos \left(\theta_{i}+\psi_{x_{i}}\right) . Z$ can be decomposed as [11]

$$
Z=\sqrt{B} G \text {, }
$$

where

$$
\begin{gathered}
B \sim \mathcal{S}\left(\mu_{B}=\frac{2}{\alpha}, \beta_{B}=1, \eta_{B}=\cos \left(\frac{\pi}{\alpha}\right)\right) \\
G \sim \mathcal{C N}\left(0, P_{I} \nu\right), \nu=4 e^{\sigma^{2} / \alpha}\left(\pi \lambda C_{4 / \alpha}^{-1} \mathbb{E}\left[\left|\xi_{i}\right|^{4 / \alpha}\right]\right)^{\alpha / 2} .
\end{gathered}
$$

Thus, conditioned on $B, Z+W \sim \mathcal{C N}\left(0, P_{I} \nu B+N_{0}\right)$. For the particular case of $h_{x_{i}} \sim \mathcal{C N}(0,1)$, i.e., Rayleigh fading, $\mathbb{E}\left[\left|\xi_{i}\right|^{4 / \alpha}\right]$ can be easily computed. Since conditioned on $s_{i}$, $\xi_{i}=\left[\left|h_{x_{i}}\right| a_{i} \cos \left(\theta_{i}+\psi_{x_{i}}\right)\right] \sim \mathcal{N}\left(0, a_{i}^{2} / 2\right)$, we have,

$$
\mathbb{E}\left[\left|\xi_{i}\right|^{4 / \alpha}\right]=\frac{\Gamma(1 / 2+2 / \alpha)}{\sqrt{\pi}} \mathbb{E}\left[a_{i}^{4 / \alpha}\right] .
$$

In the presence of channel state information (CSI) of the $S-D$ link only, the receiver employs simple coherent demodulation. The conditional error probability is thus given by the error expression for coherent detection in Gaussian noise, denoted by $h(\gamma)$ (for example, $h(\gamma)=Q(\sqrt{2 \gamma})$ for binary phase shift keying (BPSK) modulation) with

$$
\gamma=\frac{\bar{P}_{0} e^{\sigma g_{x_{s}}}\left|h_{x_{s}}\right|^{2} r_{0}^{-\alpha}}{\bar{P}_{I} \nu B+1},
$$

where $\bar{P}_{0}=P_{0} / N_{0}$ and $\bar{P}_{I}=P_{I} / N_{0}$ are the noise normalized transmit powers of the node $S$ and the interfering nodes, respectively. $\bar{P}_{0}$ and $\bar{P}_{I}$ are referred to as signal-to-noise ratio (SNR) and interference-to-noise ratio (INR), respectively. In interference limited scenario, $\gamma$ can be expressed as $\gamma=\rho X$, where $\rho=\bar{P}_{0} e^{\sigma g_{x_{s}}} r_{0}^{-\alpha} /\left(\bar{P}_{I} \nu\right)$ is a non-random quantity, referred to as SIR, and $X=\left|h_{x_{s}}\right|^{2} / B$ is a RV. Note that for the fixed $S-D$ link, the shadowing coefficient $g_{x_{s}}$ is assumed to remain constant.

\footnotetext{
${ }^{1}$ The real and imaginary components of a CS comple stable RV $\mathcal{S}_{c}(\mu, \beta=$ $0, \eta)$ are both $\mathcal{S}(\mu, \beta=0, \eta)$.
} 


\section{B. Average error probability}

The average probability of error can be expressed as

$$
P_{e}=\int_{0}^{\infty} h(\rho x) f_{X}(x) d x
$$

where $f_{X}(x)$ is the probability density function (PDF) of $X$. Since the PDF is not available in closed form, which is the main obstacle in deriving the closed-form expression for (10), we exploit the Mellin transform (MT) information of $h(\gamma)$ and $f_{X}(x)$ to derive UA for (10). UA is a rational function that matches the asymptotics of $P_{e}(\rho)$ as $\rho \rightarrow 0$ and $\rho \rightarrow \infty$, simultaneously.

The MT of $f_{X}(x)$ is given by

$$
\mathcal{F}_{X}(s)=\mathcal{F}_{\left|h_{x_{d}}\right|^{2}}(s) \mathcal{F}_{B}(2-s),
$$

where $\mathcal{F}_{\left|h_{x_{d}}\right|^{2}}(s)$ and $\mathcal{F}_{B}(s)$ are the MTs of the PDFs of $\left|h_{x_{d}}\right|^{2}$ and $B$, respectively. The MT of the stable RV $B$ with parameters given in (6) is given by [18]

$$
\mathcal{F}_{B}(s)=\frac{\Gamma\left(1+\frac{\alpha}{2}-\frac{\alpha}{2} s\right)}{\Gamma(2-s)} .
$$

If the MTs $\mathcal{F}_{\left|h_{0}\right|^{2}}(s)$ and $\mathcal{H}(s)$ are known and they have only first order poles, the UA can be easily derived by using [15, Eqn. (14)] for any linear modulation and any fading model with the required coefficients computed according to [15, Proposition 2].

1) Performance with BPSK modulation in Rayleigh fading: In the following example, we consider the case where the transmitter $S$ employs BPSK modulation, and the $S-D$ link is Rayleigh faded. In this case, $\mathcal{F}_{X}(1-s)=\Gamma\left(1-\frac{\alpha}{2} s\right)$ and $\mathcal{H}(s)=\frac{\Gamma(s+1 / 2)}{2 s \sqrt{\pi}}$. We can observe that $F_{X}(1-s)$ has poles at $s=2 / \alpha, 4 / \alpha, 6 / \alpha, \ldots$, while $\mathcal{H}(s)$ has poles at $s=0,-1 / 2,-3 / 2, \ldots$. The first positive pole at $s=2 / \alpha$ indicates that the diversity order is $2 / \alpha$. The diversity order thus, depends on the power loss exponent $\alpha$. The required coefficients $b(0)$ and $c(l)$ to compute the UA can be obtained by using [15, Proposition 2].

Example: For $\alpha=4$, we have $\tau=1 / 2, \delta=1$, and the required coefficients $b(0)$ and $c(l)$ are given by

$$
b(0)=\frac{1}{2 \sqrt{\pi}}, \quad c(l)= \begin{cases}\frac{1}{2} & l=0 \\ \frac{(-1)^{(l+1) / 2} \Gamma[l]}{\sqrt{\pi} \Gamma[(l+1) / 2]} & l=1,3, \ldots \\ 0 & \text { otherwise. }\end{cases}
$$

The UA can be readily computed by using [15, Eqn. (14)].

The average bit error rate (BER) of the $S-D$ link when $S$ as well as all the interfering nodes employ BPSK modulation, and all the links (desired and interfering) undergo independent Rayleigh fading is depicted in Fig. 1 for different densities of interfering nodes, $0.1 \mathrm{Km}^{-2}, 0.4 \mathrm{Km}^{-2}$ and $1 \mathrm{Km}^{-2}$. Both UA and Monte Carlo simulation results are plotted against the SNR. The parameters used are $\alpha=4, r_{0}=1 \mathrm{Km}$, $g_{x_{s}}=0$ (no shadowing on the desired link), INR $=20 \mathrm{~dB}$, $\sigma_{\mathrm{dB}}=10$, i.e., $\sigma=\ln 10$. Note that noise is not ignored in the simulation results. The excellent match between the UAs and

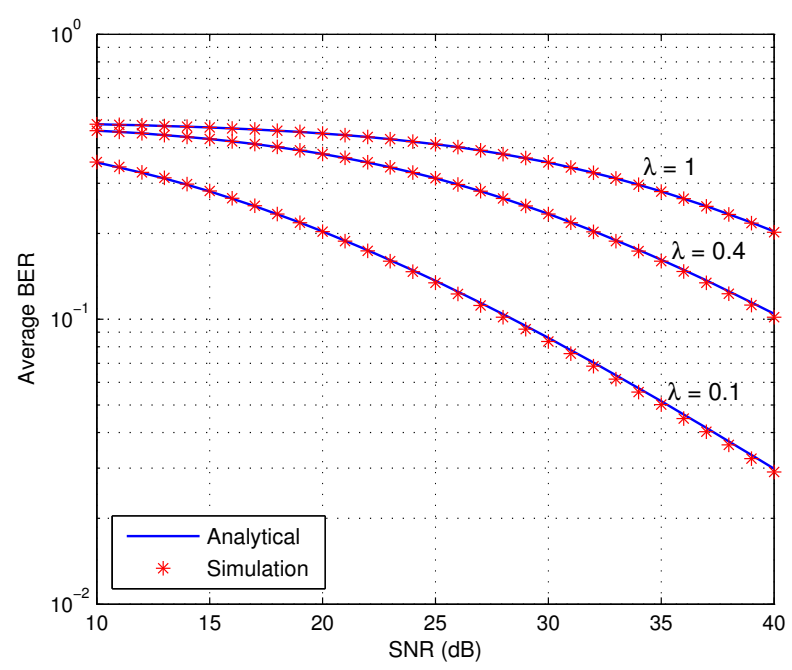

Fig. 1. Average BER of $S-D$ link with BPSK modulation for different densities of interfering nodes $\left(\alpha=4, r_{0}=1 \mathrm{Km}, g_{x_{d}}=0 \mathrm{INR}=20 \mathrm{~dB}\right.$, $\left.\sigma_{\mathrm{dB}}=10\right)$

the simulated results shows thats the error performance can be accurately evaluated with UA method in interference limited scenario. We can observe that the error performance improves with the decrease in node density due to the decrease in total interference power.

2) Performance with $N_{r}$-branch $M R C$ receiver in all Rayleigh fading: Let $N_{r}$ antennas are employed at the receiver $D$ with MRC. If $\left\{h_{x_{s}, l}=\left|h_{x_{s}, l}\right| \exp \left(j \psi_{x_{s}, l}\right), l=\right.$ $\left.1,2, \ldots, N_{r}\right\}$ are i.i.d. fading coefficients from $S$ to each receive antenna of $D$, and similarly, $\left\{h_{x_{i}, l}=\right.$ $\left.\left|h_{x_{i}, l}\right| \exp \left(j \psi_{x_{i}, l}\right), l=1,2, \ldots, N_{r}\right\}$ are i.i.d. fading coefficients from $i$ th interferer, the resultant signal after combining the received signals at each antenna can be expressed as

$$
Y=\sqrt{P_{0}} \frac{e^{\frac{\sigma}{2} g_{x_{s}}} \sum_{l=1}^{N_{r}}\left|h_{x_{s}, l}\right|^{2}}{r_{0}^{\alpha / 2}} s_{0}+Z+W,
$$

where

$$
Z=\sqrt{P_{I}} \sum_{x_{i} \in \Phi} \frac{e^{\frac{\sigma}{2} g_{x_{i}}} \sum_{l=1}^{N_{r}} h_{x_{s}, l}^{*} h_{x_{i}, l}}{r_{i}^{\alpha / 2}} s_{i}
$$

is the resultant interference, and $W=\sum_{l=1}^{N_{r}} h_{x_{s}, l}^{*} n_{l}$ is the resultant noise. Since $\left\{n_{l} \sim \mathcal{C N}\left(0, N_{0}\right), l=1,2, \ldots, N_{r}\right\}$ are i.i.d. complex Gaussian noise, conditioned on $\left\{h_{x_{s}, l}\right\}, W \sim$ $\mathcal{C N}\left(0, N_{0} \sum_{l=1}^{N_{r}}\left|h_{x_{d}, l}\right|^{2}\right)$. $Z$ can again shown to be a CS complex stable RV, which can be decomposed according to (5) with $B$ and $G$ given by (6) and (7), respectively. But $\xi_{i}$ in this case is given by

$$
\xi_{i}=\sum_{l=1}^{N_{r}}\left|h_{x_{s}, l}\right|\left|h_{x_{i}, l}\right| a_{i} \cos \left(\theta_{i}+\psi_{x_{i}, l}-\psi_{x_{d}, l}\right) .
$$

Thus, conditioned on $B$ and $\left\{h_{x_{s}, l}\right\}, Z+W \sim \mathcal{C N}\left(0, P_{I} \nu B+\right.$ $\left.N_{0} \sum_{l=1}^{N_{r}}\left|h_{x_{s}, l}\right|^{2}\right)$. 
If the interference links are Rayleigh faded, then conditioned on $\left\{h_{x_{s}, l}\right\}$,

$$
\mathbb{E}\left[\left|\xi_{i}\right|^{4 / \alpha}\right]=\frac{\Gamma(1 / 2+2 / \alpha)}{\sqrt{\pi}} \mathbb{E}\left[a_{i}^{4 / \alpha}\right] \sum_{l=1}^{N_{r}}\left|h_{x_{s}, l}\right|^{2} .
$$

In interference limited scenario, $\gamma$ can again be expressed as $\gamma=\rho X$, where

$$
\rho=\frac{\bar{P}_{0} e^{\sigma g_{x_{s}}} r_{0}^{-\alpha}}{\bar{P}_{I} \kappa}, X=\frac{\sum_{l=1}^{N_{r}}\left|h_{x_{s}, l}\right|^{2}}{B},
$$

$\kappa=4 e^{\sigma^{2} / \alpha}\left(\sqrt{\pi} \lambda C_{4 / \alpha}^{-1} \Gamma\left(\frac{1}{2}+\frac{2}{\alpha}\right) \mathbb{E}\left[a_{i}^{4 / \alpha}\right]\right)^{\alpha / 2}$. The error probability UA can be similarly obtained as in the singleantenna receiver case with the help of MT information of $h(\gamma)$ and $f_{X}(x)$.

If $\left\{h_{x_{s}, l}, l=1,2, \ldots, N_{r}\right\}$ undergo i.i.d. Rayleigh fading, then

$$
\mathcal{F}_{X}(1-s)=\frac{\Gamma\left(N_{r}-s\right) \Gamma\left(1-\frac{\alpha}{2} s\right)}{\left(N_{r}-1\right) ! \Gamma(1-s)}
$$

which have poles at $s=2 / \alpha, 4 / \alpha, 6 / \alpha, \ldots$, and at $s=$ $N_{r}, N_{r}+1, N_{r}+2, \ldots$, along with zeros at $s=1,2,3, \ldots$. For exponentially decaying $h(\gamma)$ as $\gamma \rightarrow \infty, \mathcal{H}(s)$ has only negative poles. The first positive pole at $s=2 / \alpha$ thus indicates that the diversity order is given by $2 / \alpha$, irrespective of the number of antennas at the receiver $D$.

The required parameters to compute UA for the average error probability of BPSK modulation for $\alpha=4$ are $\tau=1 / 2$, $\delta=1$ and

$$
\begin{aligned}
b(0) & =\frac{\Gamma\left(N_{r}-1 / 2\right)}{2 \pi \Gamma\left(N_{r}\right)}, \\
c(l) & = \begin{cases}\frac{1}{2} & l=0 \\
\frac{(-1)^{(l+1) / 2} \Gamma\left(N_{r}+l / 2\right) \Gamma(l)}{\sqrt{\pi} \Gamma((l+1) / 2) \Gamma\left(N_{r}\right) \Gamma(l / 2+1)} & l=1,3, \ldots \\
0 & \text { otherwise. }\end{cases}
\end{aligned}
$$

Note that when $\alpha=4$, the poles at $s=1,2,3, \ldots$ are canceled by the zeros and the effective poles of $\mathcal{F}_{X}(1-s)$ are $s=$ $1 / 2,3 / 2, \ldots$ and $s=N_{r}, N_{r}+1, N_{r}+2, \ldots$.

UA and simulation result for the average BER in all Rayleigh fading scenario with BPSK modulation at each node are plotted against SNR in Fig. 2 for different values of $N_{r}$. It is clearly visible from the figure that diversity order of the system is the same for each value of $N_{r}$. However, the error performance improves by having more receive antennas.

\section{Average Error Probability of Downlink Cellular Networks}

While the above analysis is suitable for evaluating the average error probability of a particular user whose distance from the serving node is known, in this section, we are interested in evaluating the average error probability of any randomly selected user in a cellular network. We consider a single-tier downlink cellular network with frequency reuse

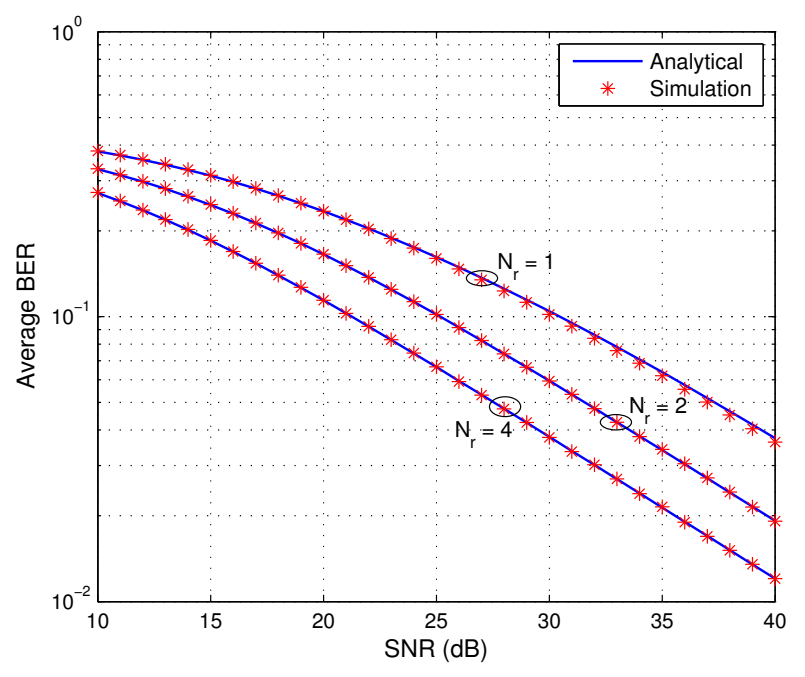

Fig. 2. Average BER of BPSK modulation with $N_{r}$-branch MRC receiver in Rayleigh fading $\left(\alpha=4, r_{0}=1 \mathrm{Km}, g_{x_{d}}=0, \lambda=0.4 \mathrm{Km}^{-2}, \mathrm{INR}=10\right.$ $\left.\mathrm{dB}, \sigma_{\mathrm{dB}}=10\right)$

1. Extension to multi-tier heterogeneous networks will be considered in future work.

\section{A. System model and error probability analysis}

The BSs are spatially distributed according a homogeneous PPP $\Phi=\left\{x_{1}, x_{2}, x_{3}, \ldots\right\}$ on $\mathbb{R}^{2}$ of intensity $\lambda$, each employing the same modulation scheme and transmitting with the same power $P$. Rayleigh fading with power law path loss is assumed between any transmitter-receiver pair. Each user in the network is associated with the BS offering the maximum received power. A typical user $o$ at the origin is thus associated with a BS located at $x_{b}=\arg \max _{x_{i} \in \Phi}\left|h_{x_{i}}\right|^{2} r_{i}^{-\alpha}$, where $r_{i}=\left\|x_{i}-o\right\|$ is the distance from $o$ to $i$ th BS and $h_{x_{i}}$ is the fading coefficient of the channel between $o$ and the $i$ th BS. The BSs other than $x_{b}$ is the set of interfering nodes for the user $o$, and is still a homogeneous PPP of intensity $\lambda$ because for a homogeneous PPP, the reduced Palm distribution is equal to the distribution of PPP itself [19].

We consider interference-limited scenario and analyze error probability at the user $o$, which is valid for any randomly chosen user according to Slivnyak's theorem [19]. By using the mathematical framework outlined in section (II-A), the conditional error probability is given by $h(\gamma)$ with

$$
\gamma=\frac{1}{\nu} \frac{\max _{i \in \Phi}\left|h_{i}\right|^{2} r_{i}^{-\alpha}}{B},
$$

where $\nu=4\left(\sqrt{\pi} \lambda C_{4 / \alpha}^{-1} \Gamma\left(\frac{1}{2}+\frac{2}{\alpha}\right) \mathbb{E}\left[a_{i}^{4 / \alpha}\right]\right)^{\alpha / 2}$, and the RV $B$ is given by (6). Let $V=\max _{x_{i} \in \Phi}\left|h_{x_{i}}\right|^{2} r_{i}^{-\alpha}$. The cumulative distribution function (CDF) of $V$ can be derived as

$$
\begin{aligned}
F_{V}(v) & =\mathbb{P}\left(\max _{x_{i} \in \Phi} h_{x_{i}} r_{i}^{-\alpha}<v\right) \\
& =\mathbb{E}_{\Phi}\left[\prod_{x_{i} \in \Phi}\left(1-\exp \left(-v r_{i}^{\alpha}\right)\right)\right] .
\end{aligned}
$$


By using the probability generating functional (PGF) of PPP [19], we have

$$
\begin{aligned}
F_{V}(v) & =\exp \left(-2 \pi \lambda \int_{0}^{\infty} \exp \left(-v r^{\alpha}\right) r d r\right) \\
& =\exp \left(-\frac{\pi \lambda}{v^{2 / \alpha}} \Gamma\left(\frac{2}{\alpha}+1\right)\right) .
\end{aligned}
$$

The corresponding PDF of $V$ can thus be obtained as

$$
f_{V}(v)=\frac{2 \pi \lambda}{\alpha} \Gamma\left(\frac{2}{\alpha}+1\right) v^{-2 / \alpha-1} e^{-\pi \lambda \Gamma\left(\frac{2}{\alpha}+1\right) v^{-2 / \alpha}} .
$$

The RV $V$ can be expressed as $V=(\pi \lambda \Gamma(2 / \alpha+$ 1) $)^{\alpha / 2} U$, where the PDF of $U$ is given by $f_{U}(u)=$ $\frac{2}{\alpha} u^{-2 / \alpha-1} \exp \left(-u^{-2 / \alpha}\right) \cdot \gamma$ can finally be expressed as $\gamma=$ $\rho X$, where $\rho=\frac{1}{4}\left[\frac{\sqrt{\pi} \Gamma\left(\frac{2}{\alpha}+1\right)}{C_{4 / \alpha}^{-1} \Gamma\left(\frac{1}{2}+\frac{2}{\alpha}\right) \mathbb{E}\left[a_{i}^{4 / \alpha}\right]}\right]^{\alpha / 2}$ and $X=\frac{U}{B}$.

The average error probability is then given by (10). If we closely observe $\rho$ and $X$, we can see that in interference limited cellular networks, the average error probability is independent of the BS density $\lambda$ and the SNR $\bar{P}=P / N_{0}$. Thus, error probability can not be improved by increasing SNR because this would increase the interference power as well. On the other hand, if we increase $\lambda$, the gain in desired received signal power due to closer distance between the user and the serving BS is counter balanced by the increase in interference power. However, by increasing $\lambda$, more users can be simultaneously served and the network capacity naturally improves without affecting the error performance of the network.

Unlike $\rho$ in section II, it is now the function of $\alpha$ and $a_{i}$ only. For the typical values of $\alpha$ in the range $2<\alpha \leq 6$ and $\mathbb{E}\left[a_{i}^{2}\right]=1$, the range of $\rho$ is much limited compared to the range $0<\rho<\infty$ in section II. Thus, the UA approach which basically matches the high-SIR $(\rho \rightarrow \infty)$ and low-SIR $(\rho \rightarrow 0)$ asymptotics may not make much sense in this case. However, one can still exploit MT information of $f_{X}(x)$ and $h(\gamma)$ to evaluate $(10)$.

Equation (10) can be transformed via Parseval formula for MT [20] as

$$
P_{e}=\frac{1}{2 \pi j} \int_{c-j \infty}^{c+j \infty} \frac{1}{\rho^{s}} \mathcal{H}(s) \mathcal{F}_{X}(1-s) d s,
$$

where $c$ lies in the fundamental strip of both $\mathcal{H}(s)$ and $\mathcal{F}_{X}(1-$ $s)$. The MT $\mathcal{F}_{X}(s)$ is given by $\mathcal{F}_{X}(s)=\mathcal{F}_{U}(s) \mathcal{F}_{B}(2-s)$, where $\mathcal{F}_{U}(s)$ can be derived as

$$
\mathcal{F}_{U}(s)=\int_{0}^{\infty} u^{s-1} f_{U}(u) d u=\Gamma\left(-\frac{\alpha}{2} s+\frac{\alpha}{2}+1\right),
$$

and $\mathcal{F}_{B}(s)$ is given by (12). We can apply residue theorem to obtain a series representation of (23), which can often be expressed in terms of generalized Hypergeometric function ${ }_{p} F_{q}()$ [21], thus yielding closed-form expression.

\section{B. Average error probability in BPSK modulation}

For BPSK modulation, (23) can be expressed as

$$
P_{e}=\frac{1}{2 \pi j} \int_{c-j \infty}^{c+j \infty} \frac{\Gamma(s+1 / 2)}{2 s \sqrt{\pi}} \frac{\Gamma\left(\frac{\alpha}{2} s+1\right) \Gamma\left(1-\frac{\alpha}{2} s\right)}{\Gamma(1-s)} \rho^{-s} d s .
$$

The poles to the left of the contour $\mathcal{R}(s)=c$ are $s=0,-(k+$ $1 / 2),-2(k+1) / \alpha$, where $k=0,1,2, \ldots$. For $\alpha \neq 4$ in the range $2<\alpha \leq 6$, the poles are simple. Thus, by closing the contour to the left, and then applying residue theorem, we have

$$
\begin{aligned}
& P_{e}=\frac{1}{2}+\sum_{k=0}^{\infty} \frac{(-1)^{k+1} \Gamma\left(-\frac{2}{\alpha}+\frac{1}{2}-\frac{2}{\alpha} k\right) \Gamma(2+k)}{k !(k+1) 2 \sqrt{\pi} \Gamma\left(\frac{2}{\alpha}+1+\frac{2}{\alpha} k\right)} \rho^{\frac{2(k+1)}{\alpha}} \\
& +\sum_{k=0}^{\infty} \frac{(-1)^{k+1} \Gamma\left(-\frac{\alpha}{4}+1-\frac{\alpha}{2} k\right) \Gamma\left(\frac{\alpha}{4}+1+\frac{\alpha}{2} k\right)}{k !\left(k+\frac{1}{2}\right) 2 \sqrt{\pi} \Gamma\left(\frac{3}{2}+k\right)} \rho^{k+\frac{1}{2}}
\end{aligned}
$$

for $\alpha \neq 4$. The infinite series can be expressed in terms of Hypergeometric function as

$$
\begin{aligned}
P_{e}= & \frac{1}{2}+\sum_{r=1}^{d} \frac{(-1)^{r} \alpha}{4 \sqrt{\pi}} \frac{\Gamma\left(-\frac{\alpha}{4}(2 r-1)+1\right) \Gamma\left(\frac{\alpha}{4}(2 r-1)\right)}{\Gamma(r) \Gamma\left(r+\frac{1}{2}\right)} \rho^{r-\frac{1}{2}} \\
\times{ }_{1} F_{2 d} & {\left[1 ; \frac{r+1 / 2}{d}, \frac{r+1 / 2}{d}+\frac{1}{d}, \ldots, \frac{r+1 / 2}{d}+\frac{d-1}{d},\right.} \\
& \left.\quad \frac{r}{d}, \frac{r}{d}+\frac{1}{d}, \ldots, \frac{r}{d}+\frac{d-1}{d} ; \frac{(-1)^{(c+d)} \rho^{d}}{d^{2 d}}\right] \\
+ & \sum_{r=1}^{c} \frac{(-1)^{r}}{2 \sqrt{\pi}} \frac{\Gamma\left(-\frac{2}{\alpha} r+\frac{1}{2}\right)}{\Gamma\left(\frac{2}{\alpha} r+1\right)} \rho^{\frac{2}{\alpha}}{ }_{1} F_{2 d}\left[1 ; \frac{r}{c}+\frac{1}{d}, \ldots, \frac{r}{c}+\frac{d}{d},\right. \\
& \left.\frac{r}{c}-\frac{1}{2 d}+\frac{1}{d}, \ldots, \frac{r}{c}-\frac{1}{2 d}+\frac{d}{d} ; \frac{(-1)^{(c+d)} \rho^{d}}{d^{2 d}}\right], \quad \text { (27) }
\end{aligned}
$$

where $\alpha / 2=c / d, c$ and $d$ are integers. For example, for $\alpha=2.8, c=7$ and $d=5$. The details of the proof are omitted for the sake of brevity.

For $\alpha=4$, (25) has double poles at $s=$ $-1 / 2,-3 / 2,-5 / 2, \ldots$ to the left of the contour $\mathcal{R}(s)=c$. In order to avoid residue computation with double poles, (25) is expressed as follows for $\alpha=4$ by substituting $s=-s$ and utilizing the properties of Gamma function [21]:

$P_{e}=-\frac{1}{2 \pi j} \int_{-c-j \infty}^{-c+j \infty} \frac{\Gamma\left(-s+\frac{1}{2}\right) \Gamma(-2 s+1) \Gamma\left(s+\frac{1}{2}\right)}{2^{1-2 s} \pi s} \rho^{s} d s$

which then has simple poles at $s=-1 / 2,-3 / 2,-5 / 2, \ldots$ to the left of the contour $\mathcal{R}(s)=-c$. Again by applying residue theorem, followed by identifying the series with ${ }_{p} F_{q}()$, the average error rate for $\alpha=4$ can finally be expressed as

$$
P_{e}=\frac{1}{2 \pi \sqrt{\rho}}{ }_{3} F_{0}\left[1,1, \frac{1}{2} ;-\frac{1}{\rho}\right] .
$$

In Fig. 3, the simulated BER of a typical user is plotted against SNR without ignoring the impact of noise for three different BS densities $0.01 \mathrm{BS} / \mathrm{Km}^{2}, 0.1 \mathrm{BS} / \mathrm{Km}^{2}$ and $0.4 \mathrm{BS} / \mathrm{Km}^{2}$. Our analytical result based on interference limited assumption is also plotted in the figure which is independent of both $\lambda$ and SNR. We can observe that the simulation and analytical results converge once the network become interference limited. If the value of $\lambda$ is large, which is expected in future networks for higher capacity, the network becomes interference limited even at smaller values of SNR.

The impact of power loss exponent, $\alpha$ in the average BER of downlink cellular network is assessed in Fig. 4. The 


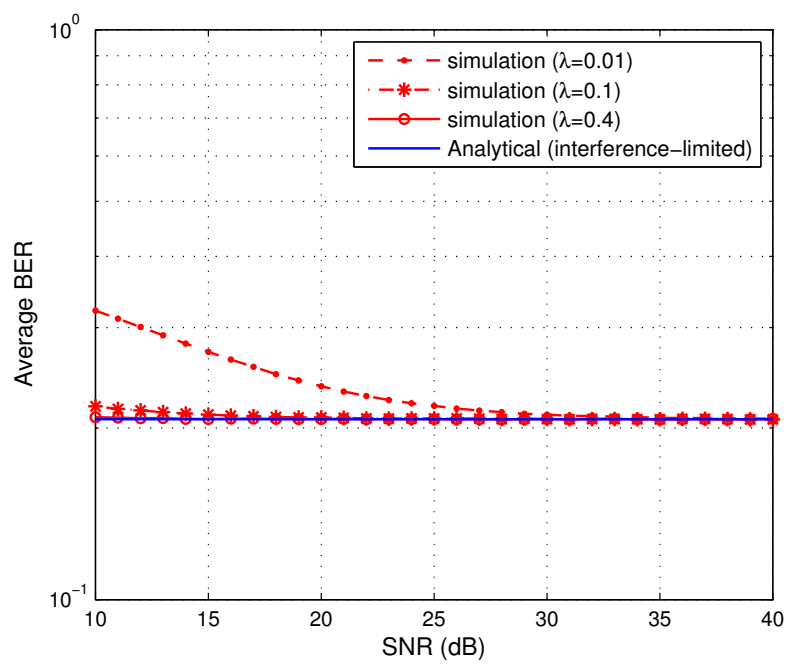

Fig. 3. Average BER of a typical user in a downlink cellular network with BPSK modulation $(\alpha=3)$

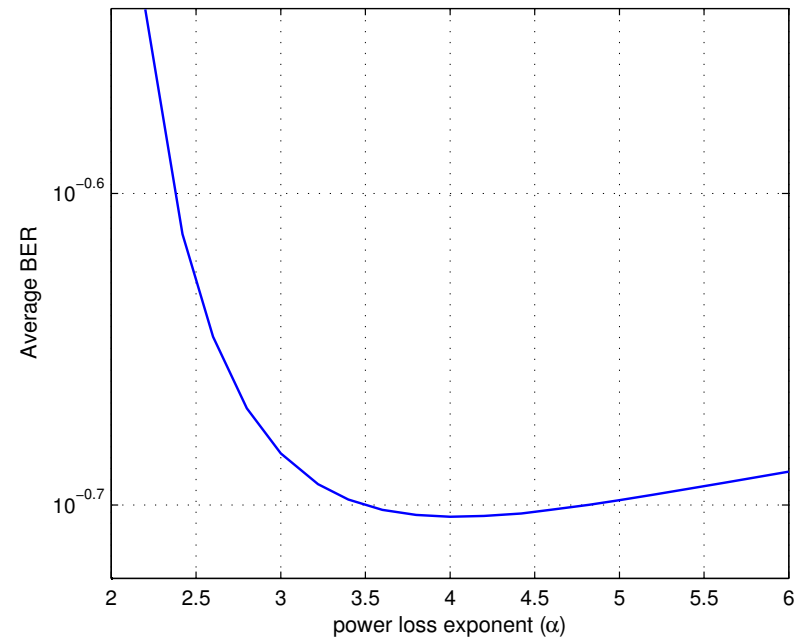

Fig. 4. Average BER versus power loss exponent $(\alpha)$ in downlink cellular network

error rate initially drops with the increase in $\alpha$ due to the decrease in aggregate interference power received at a typical user. However, the received power from the serving BS also decreases at the same time. Eventually, when the decrease in desired signal power due to increase in $\alpha$ becomes more significant compared to the decrease in aggregate interference power, the error rate starts increasing.

\section{CONCLUSION}

The contributions of the paper are two-fold:

1) We developed UA for error probability of an intended link subject to interference from surrounding nodes distributed according to a PPP. Both single-antenna and $M R C$ receivers are analyzed. The diversity order of the system is found to be $2 / \alpha$, independent of the number of receive antennas.

2) We introduced MT based approach to error probability analysis of a typical user in cellular network, which is served by the BS providing the maximum received power. The BSs are distributed according to a PPP. A closed-form expression for the average BER of BPSK modulation in Rayleigh fading is derived. The average error probability is found to be independent of SNR and BS density.

\section{REFERENCES}

[1] F. Baccelli and B. Blaszczyszyn, Stochastic Geometry and Wireless Networks. NOW: Foundations and Trends in Networking, 2010.

[2] M. Haenggi and R. K. Ganti, Interference in Large Wireless Networks. NOW: Foundations and Trends in Networking, 2009.

[3] M. Haenggi, Stochastic Geometry for Wireless Networks. Cambridge University Press, 2013.

[4] H. S. Dhillon, R. K. Ganti, F. Baccelli, and J. G. Andrews, "Modeling and analysis of K-tier downlink heterogeneous cellular networks," IEEE J. Sel. areas in Commun., vol. 30, no. 3, pp. 550-560, Apr. 2012.

[5] S. Mukherjee, "Distribution of downlink SINR in heterogeneous cellular networks," IEEE J. Sel. Areas Commun., vol. 30, no. 3, pp. 54-64, Apr. 2012.

[6] H.-S. Jo, Y. J. Sang, P. Xia, and J. G. Andrews, "Heterogeneous cellular networks with flexible cell association: A comprehensive downlink SINR analysis." IEEE Trans. Wireless Commun., vol. 11, no. 10, pp. 34843495, Oct. 2012.

[7] H. ElSawy and E. Hossain, "Two-tier hetnets with cognitive femtocells: Downlink performance modeling and analysis in a multichannel environment," IEEE Trans. on Mobile Computing, vol. 13, no. 3, pp. 649-663, Mar. 2014.

[8] Y. Jin and L. Qiu, "Joint user association and interference coordination in heterogeneous cellular networks," IEEE Commun. Letters, vol. 17, no. 12, pp. 2296-2299, Dec. 2013.

[9] J. G. Andrews, F. Baccelli, and R. K. Ganti, "A tractable approach to coverage and rate in cellular networks," IEEE Trans. Commun., vol. 59, no. 11, pp. 3122-3134, Nov. 2011

[10] M. Z. Win, P. C. Pinto, and L. A. Shepp, "A mathematical theory of network interference and its applications," IEEE Proc., vol. 97, no. 2, pp. 205-230, Feb. 2009.

[11] P. C. Pinto and M. Z. Win, "Communication in a Poisson field of interferers-part i: Interference distribution and error probability," IEEE Trans. Wireless Commun., vol. 9, no. 7, pp. 2176-2186, Jul. 2010.

[12] C. Merola, A. Guidotti, M. Di Renzo, F. Santucci, and G. Corazza, "Average symbol error probability in the presence of network interference and noise," in Proc. IEEE Int. Conf. on Commun. (ICC), Ottawa, Canada, Jun. 2012, pp. 2613-2618

[13] M. D. Renzo, C. Merola, A. Guidotti, F. Santucci, and G. E. Corazza, "Error performance of multi-antenna receivers in a Poisson field of interferers: A stochastic geometry approach," IEEE Trans. Commun., vol. 61, no. 5, pp. 2025-2047, May 2013.

[14] C. Tellambura, Y. Dhungana, and M. Soysa, "Uniform approximations for wireless performance in fading, noise and interference," in Proc. IEEE Int. Conf. on Commun. (ICC), Ottawa, Canada, Jun. 2012, pp. 2410-2415.

[15] Y. Dhungana and C. Tellambura, "Uniform approximations for wireless performance in fading channels," IEEE Trans. Commun., vol. 16, no. 11, pp. 4768-4779, Nov. 2013.

[16] P. Guan and M. D. Renzo, "Stochastic geometry analysis of the average error probability of downlink cellular networks," in Proc. IEEE Int. Conf. on Computing, Networking and Commun. (ICNC)), Honolulu, HI, Feb. 2014, pp. 649-655.

[17] G. Samoradnitsky and M. Taqqu, Stable Non-Gaussian Random Processes. Chapman and Hall, 1994.

[18] V. M. Zolotarev, One-dimensional stable distributions, ser. Translations of mathematical monographs. Providence, Rhode Island: American Mathematical Society, 1986.

[19] S. N. Chiu, D. Stoyan, W. S. Kendall, and J. Mecke, Stochastic Geometry and its Applications, 3rd ed. John Wiley and Sons, 2013.

[20] G. Fikioris, "Integral evaluation using the mellin transform and generalized hypergeometric functions: Tutorial and applications to antenna problems," IEEE Trans. Antennas and Propagation, vol. 54, no. 12, pp. 3895-3907, Dec. 2006

[21] I. S. Gradshteyn and I. M. Ryzhik, Table of Integrals, Series, and Products, 7th ed. Academic Press, Inc., 2007. 